\title{
Caffeine Induces the Stress Response and Up-Regulates Heat Shock Proteins in Caenorhabditis elegans
}

\author{
Mohammad Al-Amin, Ichiro Kawasaki, Joomi Gong, and Yhong-Hee Shim*
}

\begin{abstract}
Caffeine has both positive and negative effects on physiological functions in a dose-dependent manner. C. elegans has been used as an animal model to investigate the effects of caffeine on development. Caffeine treatment at a high dose (30 mM) showed detrimental effects and caused early larval arrest. We performed a comparative proteomic analysis to investigate the mode of action of high-dose caffeine treatment in $C$. elegans and found that the stress response proteins, heat shock protein (HSP)-4 (endoplasmic reticulum [ER] chaperone), HSP-6 (mitochondrial chaperone), and HSP-16 (cytosolic chaperone), were induced and their expression was regulated at the transcriptional level. These findings suggest that high-dose caffeine intake causes a strong stress response and activates all three stress-response pathways in the worms, including the ER-, mitochondrial-, and cytosolic pathways. RNA interference of each hsp gene or in triple combination retarded growth. In addition, caffeine treatment stimulated a food-avoidance behavior (aversion phenotype), which was enhanced by RNAi depletion of the hsp-4 gene. Therefore, up-regulation of hsp genes after caffeine treatment appeared to be the major responses to alleviate stress and protect against developmental arrest.
\end{abstract}

\section{INTRODUCTION}

Caffeine is one of the most popular drugs in the world and its consumption had increased rapidly in modern society. Most of the caffeine consumed is from coffee. According to "caffeine informer" 2013 data, $2.6 \mathrm{~kg}$ coffee per capita (per person on average) is consumed in South Korea, making South Korea the $26^{\text {th }}$ top coffee consuming country in the world. An optimal dose of caffeine has many beneficial effects, such increasing attention and focus, improving athletic performance, and improving

Department of Bioscience and Biotechnology and Institute of KU Biotechnology, Konkuk University, Seoul 143-701, Korea

*Correspondence: yshim@konkuk.ac.kr

Received 25 October, 2015; revised 29 October, 2015; accepted 30 October, 2015; published online 7 January, 2016

Keywords: caffeine, C. elegans development, food-avoidance behavior, heat shock protein, proteomic analysis many other aspects of health (Reviewed in Weinberg and Bealer, 2002). In contrast, caffeine is an addictive drug and has side effects, particularly during pregnancy (Kuczkowasaki, 2009). High caffeine consumption may increase the risk for high blood pressure, hypertension, insomnia, and panic disorder (Mesas et al., 2011; Vilarim et al., 2011). High caffeine consumption also has maternal effects during pregnancy, which are related with a greater risk for low birth weight infants (Chen et al, 2014). A lethal human caffeine dose is approximately $10 \mathrm{~g}$ (one cup of coffee contains $\sim 125 \mathrm{mg}$ of caffeine) (Ekwall et al., 1998). Although high doses of caffeine can be lethal, the mode of action remains unclear.

In our previous study, we investigated effects of caffeine on Caenorhabditis elegans as an animal model organism. We showed that a high dose $(30 \mathrm{mM})$ of caffeine inhibits early larval development (Min et al., 2015), suggesting that a high dose of caffeine is a developmental stressor. Several reports have demonstrated that caffeine affects $C$. elegans development in a dose-dependent manner. That is, low-dose (5 and $10 \mathrm{mM}$ ) caffeine extends life span, whereas a high-dose (30 mM) shortens life span (Sutphin et al., 2012). To understand the molecular basis leading to the developmental stress after high-dose caffeine treatment, we performed a comparative proteomic analysis in this study. Here, we report that the heat shock proteins (HSPs), stress response proteins, were highly induced after treating $C$. elegans with $30 \mathrm{mM}$ caffeine.

\section{MATERIALS AND METHODS}

\section{Strains and maintenance of $C$. elegans}

Maintenance and handling of $C$. elegans were performed as described previously (Brenner, 1974). Strain N2 was used as the wild type for all analyses. Strains SJ4005: zcls4[hsp4::GFP], SJ4100: zcls13[hsp-6::GFP], and TJ375: gp/s1[hsp16.2::GFP] were used for the caffeine stress-induced HSP expression analysis. Worms were maintained at either $15^{\circ} \mathrm{C}$ or $20^{\circ} \mathrm{C}$ on Nematode Growth Medium (NGM) agar plates containing Escherichia coli strain OP50 supplemented with $5 \mu \mathrm{g} / \mathrm{ml}$ of cholesterol.

\section{Caffeine feeding}

Appropriate amounts of caffeine (Sigma, USA) were included in the NGM before autoclaving to obtain final concentrations of 10$30 \mathrm{mM}$. After E. coli strain OP50 was seeded on the plates, L1synchronized worms were cultured on the caffeine-containing 
NGM agar plates to determine the responses.

Two-dimensional (2-D) gel electrophoresis and matrix-assisted laser desorption/ionization time-of-flight (MALDI-TOF) mass spectrometry (MS) analyses

Synchronized L1-stage worms were cultured in NGM containing 0 or $30 \mathrm{mM}$ caffeine in a shaking incubator, and the total proteome was extracted and quantified as described previously (Ahn et al., 2006). After quantification, 2-D gel electrophoresis; that is, isoelectric focusing ( $\mathrm{p} / 3-10$ or $\mathrm{p} / 4-7$ ) and vertical sodium dodecyl sulfate gradient $(7-13 \%)$ slab gel electrophoresis were performed as described previously (Kawasaki et al., 2013). Stained gels were scanned using an ImageScanner (Amersham Bioscience, USA), and analyzed with Melanie-4 (GeneBio, Switzerland). More than two-fold differentially expressed protein spots were analyzed by MALDI-TOF MS and publicized protein functions were cited from the wormbase (http://wormbase.org).

\section{Real-time quantitative reverse transcription-polymerase} chain reaction ( $q R T-P C R)$

Synchronized L1-stage worms were grown on NGM agar plates with or without caffeine for $24 \mathrm{~h}$. Total RNA was extracted from the worms with Trizol reagent (Sigma), purified, and reverse transcribed with M-MLV reverse transcriptase (Gibco BRL, USA) using oligo-dT primers (Promega, USA) to synthesize the first-strand cDNA. Respective cDNA products were PCR-amplified using the following primers: $h s p-3(\mathrm{C} 15 \mathrm{H} 9.6) \mathrm{F}$ primer, 5'-ACCATCCAGGTCTTCGAAGG-3', and $h s p-3$ (C15H9.6) $\mathrm{R}$ primer, 5'- AACCTCAATTTGTGGAACTCCG-3', for $h s p-3$ cDNA; $h s p-4$ (F43E2.8) F primer, 5'-CATCTCGTGGAATCAA CCCT-3', and hsp-4 (F43E2.8) R primer, 5'-ACTTAGTCAT GACTCCTCCG-3', for $h s p-4$ cDNA; $h s p-6$ (C37H5.8) F primer, 5'-GGATGCTGGACAAATCTCTG-3', and $h s p-6$ (C37H5.8) R primer, 5'-ACAGCGATGATCTTATCTCCA-3', for $h s p-6$ cDNA; hsp-16.2 (Y46H3A.3) F primer, 5'-GTCACTTTACCACTATTT CCGT-3', and hsp-16.2 (Y46H3A.3) R primer, 5'-CAATCTCA GAAGACTCAGATGG-3', for $h s p-16.2$ cDNA. Primers for act-1 cDNA, which was used as an internal control, were the act-1 $\mathrm{F}$ primer, 5'-CCAGGAATTGCTGATCGTATGCAGAA-3', and the act-1 $\mathrm{R}$ primer, 5'-TGGAGAGGGAAGCGAGGATAGA-3'. All PCRs were performed in a $25 \mu$ reaction volume using Power SYBR $^{\circledR}$ Green PCR Master Mix (Applied Biosystems, USA). mRNA levels of each gene were averaged from triplicate measurements, normalized against that of act-1, and presented as a relative value using the $0 \mathrm{mM}$ caffeine mRNA level as 1 .

RNA interference (RNAi) analysis

The RNAi analysis was performed using the "RNAi-by-soaking" method, as described previously (Min et al., 2015).

\section{Image analysis}

Synchronized L1-stage worms were grown on NGM agar plates at $20^{\circ} \mathrm{C}$ with or without caffeine for $24 \mathrm{~h}$, and heat shock $\left(30^{\circ} \mathrm{C}\right.$ for $\left.6 \mathrm{~h}\right)$ was used as a positive control. Worms were immobilized with levamisole before mounting on $2 \%$ agarose pads for microscopic observation with a Zeiss Axioskop 2 MOT fluorescence microscope (Carl Zeiss, Germany). Images were acquired under the same exposure conditions. Average pixel intensity values were calculated by sampling images from several animals. We calculated the mean and maximum pixel intensity for each animal from these images using Image $\mathrm{J}$ software (http://rsb.info). At least 50 animals were examined for each strain/condition in each experiment. Each assay was repeated at least three times.
Aversion phenotype (food avoidance) assay

Synchronized L1-stage worms were placed at the center of 0,10 , and $30 \mathrm{mM}$ caffeine-containing NGM plates where a uniform $E$. coli OP50 lawn was spotted. The percentage of animals that stayed outside the OP50 lawn was scored after $24 \mathrm{~h}$.

\section{Statistical analysis}

All experiments were repeated more than three times for statistical analysis. Student's $t$-test was used to detect differences. A $P$-value $<0.05$ was considered significant.

\section{RESULTS}

\section{Proteomic analysis}

Comparative proteomic analyses were carried out by comparing proteomes extracted from worms grown with or without caffeine to determine the $C$. elegans protein profile in response to caffeine. We reported previously that high-dose caffeine (30 $\mathrm{mM}$ ) treatment inhibits $C$. elegans development and results in early larval stage developmental arrest (Min et al., 2015). Thus, proteomes were prepared from L1-stage worms grown with or without caffeine. Two-dimensional gel electrophoresis was performed at a p/ range of 3-10. Of the 656 spots analyzed 110 were more than two-fold differentially expressed. Thirty-six of the 110 spots were analyzed by MALDI-TOF. As a result, 20 proteins were up-regulated (Supplementary Table S1) and 15 were down-regulated (Supplementary Table S2). Twodimensional gel electrophoresis was also performed at a $\mathrm{p} /$ range of 4-7 for a better separation of the worm proteins in this

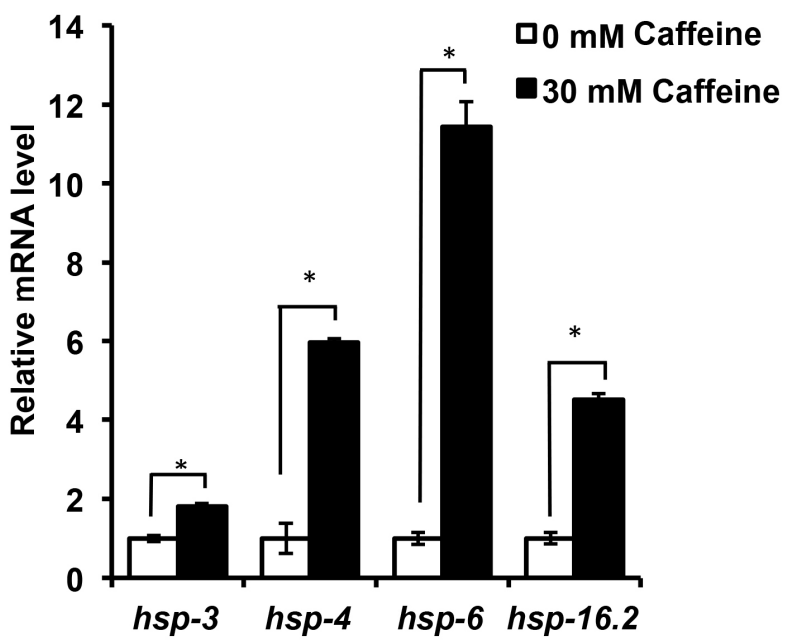

\begin{tabular}{cccc}
\hline & $\mathbf{0} \mathbf{~ m M}$ & $\mathbf{3 0} \mathbf{~ m M}$ & $\boldsymbol{P}$ value \\
\hline $\boldsymbol{h} \boldsymbol{s p - 3}$ & 1.0 & $1.79 \pm 0.08$ & 0.012 \\
$\boldsymbol{h} \boldsymbol{s p - 4}$ & 1.0 & $5.95 \pm 0.08$ & 0.002 \\
$\boldsymbol{h} \boldsymbol{s p - 6}$ & 1.0 & $11.42 \pm 0.65$ & 0.001 \\
$\boldsymbol{h} \boldsymbol{s p - 1 6 . 2}$ & 1.0 & $4.51 \pm 0.15$ & 0.001 \\
\hline
\end{tabular}

Fig. 1. Caffeine promotes transcription of heat shock protein (HSP) genes. Relative mRNA levels of $h s p$ genes were measured by qRTPCR in L1 stage larval N2 worms treated or not with $30 \mathrm{mM}$ caffeine. act-1 was used an internal control. mRNA levels are presented relative to the $0 \mathrm{mM}$ caffeine as 1.0 . 
Table 1. Differentially expressed heat shock proteins (HSPs) in worms grown with $30 \mathrm{mM}$ caffeine

\begin{tabular}{rccccc}
\hline Spot ID & Gene name & Protein identified & Theoretical mass $(\mathrm{kDa}) / \mathrm{pl}$ & Fold changed & $\%$ match \\
\hline 18471 & $h s p-4$ & HSP 70 superfamily & $76.0 . / 6.7$ & +6.79 & 18 \\
18486 & $h s p-6$ & HSP 70 superfamily & $72.3 / 5.0$ & +2.72 & 23 \\
19229 & $h s p-16.1$ & HSP 16 superfamily & $16.2 / 5.4$ & +2.25 & 76 \\
694 & $h s p-16.2$ & HSP 16 superfamily & $16.2 / 5.3$ & +2.55 & 26 \\
18433 & $h s p-3$ & HSP 70 superfamily & $73.0 / 5.0$ & -4.88 & 27 \\
\hline
\end{tabular}

range. Protein extracts were prepared in the same way as for the previous 2-D gel for p/s of 3-10. A total of 362 spots were analyzed and 74 were more than two-fold differentially expressed. Twenty-five of the 74 spots were identified by MALDITOF. As a result, 15 proteins were up-regulated (Supplementary Table S3) and eight were down-regulated (Supplementary Table S4).

HSPs were highly induced during caffeine treatment High-dose caffeine treatment is a developmental stressor because it inhibits the $C$. elegans development (Min et al., 2015). Here, HSPs were the major stress responding proteins after caffeine treatment (Table 1). HSP-4, HSP-6, and HSP-16.1 were more than two-fold up-regulated after the $30 \mathrm{mM}$ caffeine treatment when the proteome was analyzed in the $\mathrm{p} /$ range of
3-10 (Supplementary Table S1). In addition, HSP-16.2 was upregulated after analyzing the proteome in the $\mathrm{p} /$ range of 4-7 (Supplementary Table S3), whereas HSP-3 was downregulated (Supplementary Table S2). These results indicate that all three stress responding pathways were activated by caffeine including an endoplasmic reticulum (ER) chaperone (HSP-4), a mitochondrial chaperone (HSP-6), and a cytosolic chaperone (HSP-16).

Up-regulation of $h s p$ mRNA expression after the caffeine treatment was demonstrated by the qRT-PCR analysis (Fig. 1). Although HSP-4 showed the highest protein induction after caffeine treatment (6.79-fold increase), $h s p-6$ mRNA was the most up-regulated (11.42-fold increase). In contrast, HSP-3 protein expression decreased (4.88-fold decrease), even though the $h s p-3$ mRNA level increased (1.79-fold, $p=0.012$ )
$\boldsymbol{A}$

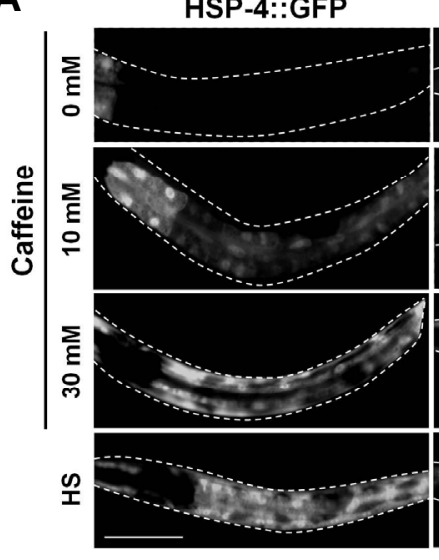

HSP-6::GFP
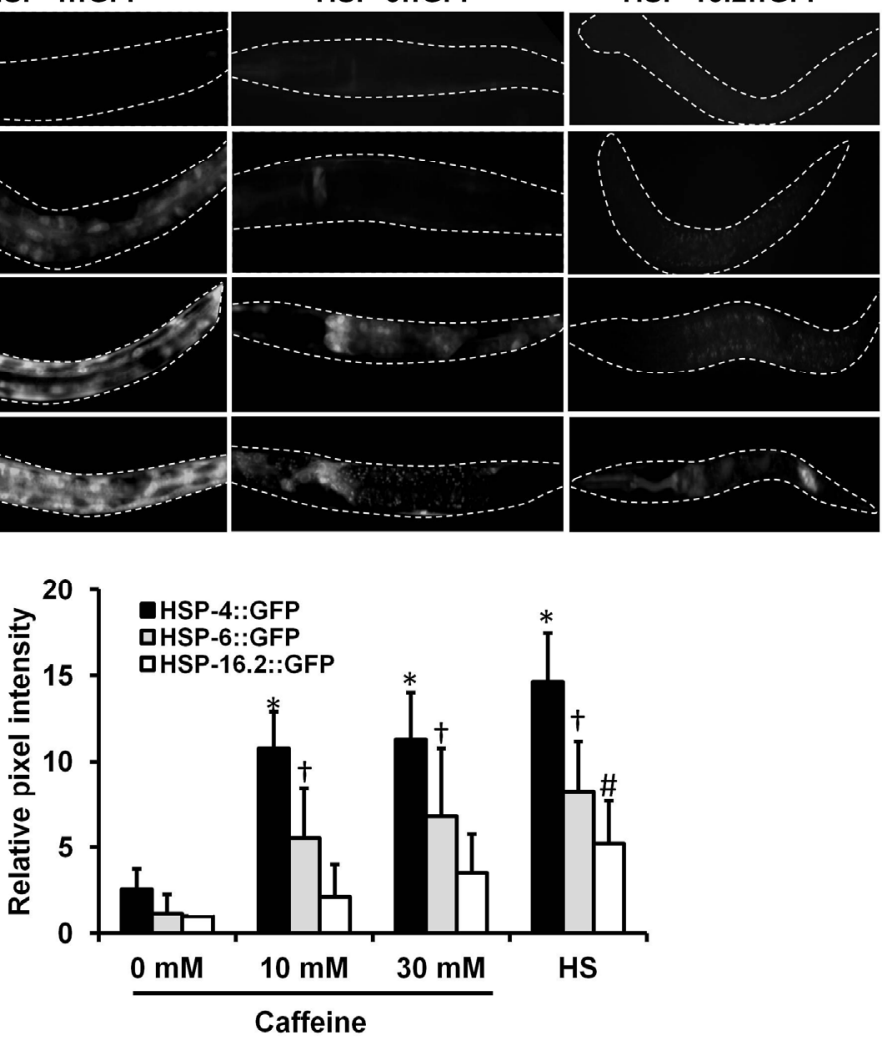

\begin{tabular}{lcrrr}
\hline Caffeine & $0 \mathrm{mM}$ & \multicolumn{1}{c}{$10 \mathrm{mM}$} & \multicolumn{1}{c}{$30 \mathrm{mM}$} & \multicolumn{1}{c}{ HS } \\
\hline HSP-4::GFP & $2.54 \pm 1.19$ & $10.77 \pm 2.12$ & $11.28 \pm 2.71$ & $14.62 \pm 2.83$ \\
HSP-6::GFP & $1.14 \pm 1.11$ & $5.51 \pm 2.94$ & $6.80 \pm 3.95$ & $8.25 \pm 2.90$ \\
HSP-16.2::GFP & $1.00 \pm 0.00$ & $2.11 \pm 1.86$ & $3.48 \pm 2.27$ & $5.20 \pm 2.47$ \\
\hline
\end{tabular}

Fig. 2. (A) Heat shock proteins (HSPs) were differently induced by caffeine treatment. Worms containing respective HSP::GFP fusion transgenes were treated with 0,10 , or $30 \mathrm{mM}$ caffeine and HSP::GFP expression at the L1 larval stage was observed under fluorescent microscopy. HS, heat shock. Scale bar, 50 $\mu \mathrm{m}$. (B) Total GFP fluorescence of each L1 worm was quantified as relative pixel intensity using Image $\mathrm{J}$ software and images taken with a CCD camera under the same exposure conditions. Relative pixel intensity was calculated by dividing the value obtained from HSP-16.2::GFP without caffeine $(0 \mathrm{mM})$, which was considered 1.0. ${ }^{*} p<0.05$ for HSP-4::GFP; $\uparrow p<0.05$ for HSP-6::GFP; \#p < 0.05 for HSP16.2::GFP 


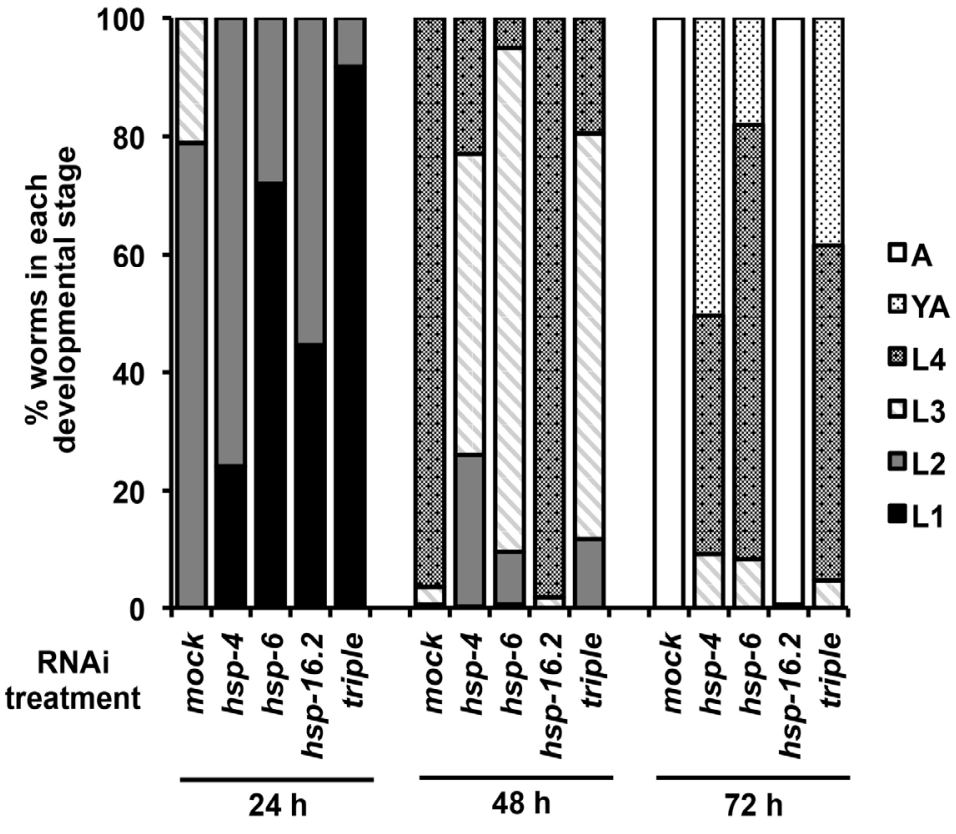

Fig. 3. Depletion of $h s p$ genes by RNAi caused growth retardation. Synchronized-L1 stage worms were treated with soaking RNAi (single or triple RNAi) to deplete $h s p$ gene activity. The developmental stages were scored at $24 \mathrm{~h}$ intervals for $72 \mathrm{~h}$, and the percent distribution of worms in each developmental stage is displayed as a percentage distribution graph for each RNAi treatment. About 400-900 individuals (triplicates of 130-300 individuals/plate) were scored for each RNAi treatment. as shown for the other $h s p$ genes. These findings suggest that HSPs in all three stress response pathways were up-regulated, primarily at the transcriptional level, after caffeine treatment.

\section{Different induction levels of the HSP-4, HSP-6, and HSP-16.2 proteins after caffeine treatment}

To confirm induction of HSPs after caffeine treatment in vivo, transgenic worms with HSP-4::green fluorescent protein (GFP), HSP-6::GFP, and HSP-16.2::GFP were examined by quantifying relative protein levels of the respective fusion proteins after caffeine treatment (Fig. 2). The transgenic worms were heat shocked to induce the proteins. As a result, caffeine induced expression of HSP-4, HSP-6, and HSP-16.2, as shown by the increase in GFP expression. The relative expression level of each protein correlated with the protein levels detected in the proteomic analysis. The highest induction level was found in HSP-4::GFP (10.77 at $10 \mathrm{mM}$ and 11.28 at $30 \mathrm{mM}$ caffeine) and the lowest was in HSP16.2::GFP (2.11 at $10 \mathrm{mM}$ and 3.48 at $30 \mathrm{mM}$ caffeine). The $30 \mathrm{mM}$ caffeine treatment resulted in the highest induction of all three HSPs compared with that of $10 \mathrm{mM}$, suggesting HSP induction by caffeine is dose-dependent.

\section{RNAi treatment of hsp genes showed the growth retardation}

Larval development was retarded when $h s p-4, h s p-6$, and $h s p-$ 16.2 were depleted by RNAi (Fig. 3). Among the three $h s p$ genes, depleting $h s p-6$ resulted in the most severe retardation. That is, $71.9 \%$ of worms remained at the L1 stage after $24 \mathrm{~h}$, whereas most of the worms in the mock RNAi control reached the L2 stage. Worms treated with $h s p-6$ RNAi were severely retarded after $72 \mathrm{~h}: 73.8 \%$ of the worms were at the L4 stage, whereas all worms in the mock RNAi control reached the adult stage (Fig. 3). Unlike $h s p-4$ and $h s p-6$ gene RNAi, $h s p-16.2$ RNAi retarded larval development only during the $L 1$ to $L 2$ stages, whereas growth of larvae in later stages was not affected (Fig. 3). These results suggest that $h s p-16.2$ has critical functions during early larval stages.
The aversion phenotype was activated by caffeine and further enhanced by RNAi of the hsp-4

The $C$. elegans aversion phenotype (food-avoidance behavior) is observed when worms sense a toxin and try to escape from the environmental stress (Melo and Ruvkun, 2012). Interestingly, the high-dose caffeine treatment stimulated this aversion phenotype (Fig. 4A). To examine whether the stress response proteins whose expression was induced by caffeine are involved in the aversion behavior, the $h s p$ genes of worms were depleted with RNAi under the condition of caffeine treatment or no treatment. We hypothesized that caffeine would induce expression of HSPs to alleviate the stress response, including aversion behavior. The level of the aversion phenotype was increased by RNAi of $h s p-4$ but not by RNAi of $h s p-6$ and $h s p$ 16.2, suggesting that HSP-4 can alleviate food-avoidance behavior of worms after caffeine treatment. Depleting the $h s p-4$ ER chaperone gene significantly increased the aversion phenotype compared to that of the $h s p-6$ mitochondrial chaperone gene or the $h s p-16.2$ cytosolic chaperone gene. RNAi of $h s p-6$ or $h s p-16.2$ showed either no effect or decreased the aversion phenotype after caffeine treatment, respectively. These findings suggest that the worms responded to caffeine primarily through an ER stress response.

\section{DISCUSSION}

Caffeine treatment has both positive and negative effects on physiological functions in a dose-dependent fashion. In general, a lower-dose results in positive effects and higher-doses result in negative effects. Therefore, understanding the mechanism of the lethal effects of high-dose caffeine treatment is important, as caffeine consumption is increasing worldwide. In this study, we performed a comparative proteomic analysis to identify caffeine-responsive proteins after treating $C$. elegans with highdose $(30 \mathrm{mM})$ caffeine. C. elegans is a valuable model organism to study the effects of caffeine at both cellular and behavioral levels. 
A

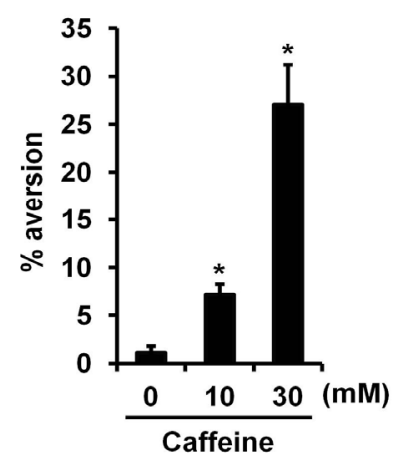

B

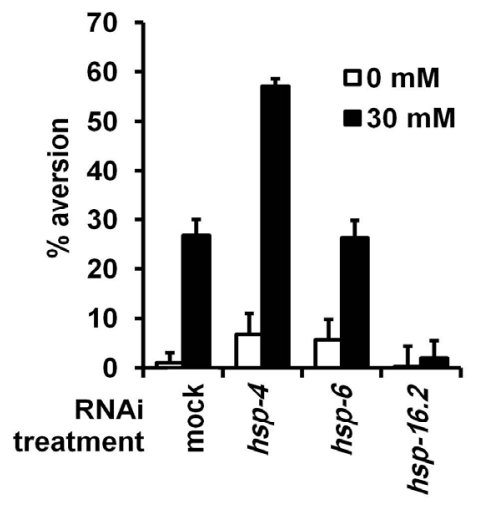

\begin{tabular}{ccc}
\hline Caffeine & $\%$ aversion & $P$ value \\
\hline $0 \mathrm{mM}$ & $1.13 \pm 0.63$ & \\
$10 \mathrm{mM}$ & $7.19 \pm 1.05$ & 0.001 \\
$30 \mathrm{mM}$ & $27.08 \pm 4.12$ & 0.004 \\
\hline
\end{tabular}

\begin{tabular}{ccc}
\hline & \multicolumn{2}{c}{$\%$ aversion } \\
\cline { 2 - 3 } & $0 \mathrm{mM}$ & \multicolumn{1}{c}{$30 \mathrm{mM}$} \\
\hline mock & $1.05 \pm 2.01$ & $26.75 \pm 3.21$ \\
$h s p-4$ & $6.76 \pm 4.24$ & $57.14 \pm 1.51$ \\
$h s p-6$ & $5.68 \pm 4.11$ & $26.25 \pm 3.54$ \\
$h s p-16.2$ & $0.29 \pm 4.12$ & $2.01 \pm 3.51$ \\
\hline
\end{tabular}

Fig. 4. The aversion phenotype was stimulated by caffeine treatment. (A) Caffeine promoted food-avoidance behavior on OP50 containing NGM spot plates. Synchronized N2 L1 larvae were cultured on $0 \mathrm{mM}(\mathrm{n}=546), 10 \mathrm{mM}(\mathrm{n}=$ $579)$, and $30 \mathrm{mM}(\mathrm{n}=988)$ caffeinecontaining plates, and the number of food-avoiding animals was counted after a $24 \mathrm{~h}$ culture. Results are shown as a percentage of food-avoiding animals from three independent experiments $\left({ }^{*} p<0.05\right)$. (B) Synchronized L1 larvae were treated with heat shock protein (hsp)-4, $h s p-6$, and $h s p-16.2$ dsRNA. After the RNAi treatment, foodavoidance behavior was observed in spot plates containing either $0 \mathrm{mM}$ ( $h s p$ 4 RNAi, $\mathrm{n}=680$; $h s p-6$ RNAi, $\mathrm{n}=299$; hsp-16.2 RNAi, $\mathrm{n}=335$ ) or $30 \mathrm{mM}$ ( $h s p-4$ RNAi, $\mathrm{n}=287$; $h s p-6$ RNAi, $\mathrm{n}=$ 259; $h s p-16.2$ RNAi, $\mathrm{n}=285$ ) of caffeine. Values are shown as percentages from two independent experiments.
Many of the proteins that were up-regulated in response to caffeine have critical functions in the developmental processes (Supplementary Table S1). RNAi depletion of them causes either larval arrest or growth defects (http://wormbase.org). Among them, expression of HSP-4, HSP-6, HSP-16.1, and HSP-16.2 increased and their depletion with RNAi retarded larval growth (Fig. 3), suggesting that these stress response proteins were induced to alleviate the negative effects of highdose caffeine that lead to larval developmental arrest. Induction of HSPs was confirmed using GFP-transgenic worms (Fig. 2). It was found that mRNA levels $h s p$ genes were also increased (Fig. 1), suggesting that HSP expression is regulated primarily at the transcriptional level after caffeine treatment. However, in case of HSP-3, its protein level was decreased by 4.88 -fold although its mRNA level was increased by 1.79 -fold after caffeine treatment. This result suggests that initially increased mRNA level of $h s p-3$ was subsequently decreased at the posttranscriptional level, which resulted in the decreased level of HSP-3 protein, or the HSP-3 protein was susceptible to protein degradation. Although an exact mechanism is not elucidated, similar discrepancy between mRNA levels and protein levels has been also reported (Kawasaki et al., 2013). HSP-4 and HSP-3 are ER stress response proteins. Interestingly, HSP-4 protein level but not that of HSP-3 increased after caffeine treatment. In fact, the HSP-3 protein level decreased, suggesting that HSP-4 is the major stress response protein in the ER stress response to caffeine. Compensatory regulation of ER chaperones has been reported previously in $C$. elegans (Kapulkin et al., 2005). That is, $h s p-4$ expression was upregulated in the absence of $h s p-3$ gene expression. Therefore, the higher increase in HSP-4 expression compared to the other HSPs may be due to reduced expression of HSP-3 after caffeine treatment. HSP-6 is a mitochondrial stress response protein (Yoneda et al., 2004) and increased HSP-6 expression after caffeine treatment indicates that caffeine also induces the mitochondrial stress pathway. Small HSPs, such as HSP-16.1 and HSP-16.2, were also upregulated after caffeine treatment. Unlike caffeine, ethanol does not induce the ER stress re- sponse, although both caffeine and ethanol induce expression of cyp genes for detoxification (Min et al., 2015; Peltonen et al., 2013). These findings suggest that the stress response to caffeine is universal among the three stress response pathways.

The balance between unfolded proteins and chaperones is regulated by the unfolded protein response (UPR) (Benedetti et al., 2006). In C. elegans, several UPR pathways are independently involved to maintain the integrity of the protein-folding in the ER, mitochondria, and cytosol. In all three compartments, each has specific chaperones to promote efficient folding. During the stress condition, specific UPR pathway is activated and sends a signal to the nucleus to induce the expression of particular chaperone genes in a compartment-specific manner (Haynes and Ron, 2010). Caffeine treatment appears to induce all three responses to activate the UPR pathways resulting in a developmental stressor. As previously reported, caffeine intake during pregnancy increases the risk of development defects in human (Chen et al, 2014), which may be due to the abnormal stress responses.

In addition, HSP-4 alleviated food-avoidance behavior (aversion phenotype) in C. elegans. The presence of the aversion phenotype after caffeine treatment suggests that high-dose caffeine was a stressor to worms and inhibited development. Interestingly, when genome-wide RNAi screening was performed previously to identify genes for the aversion phenotype, many genes essential for growth and development were detected (Melo and Ruvkun, 2012). This result supports that caffeine treatment may inactivate genes for growth and development, leading to the larval arrest and the aversion phenotype. This phenotype was enhanced by RNAi deletion of $h s p-4$ but not by RNAi of $h s p-6$ and $h s p-16.2$, suggesting that induction of $h s p-4$ gene expression activates a protective program for an animal survival by alleviating the aversion phenotype in response to caffeine. This idea is supported by the finding that a mutation in a mitochondrial gene causes the aversion phenotype and enhances $h s p-6$ gene expression (Liu et al., 2014). It remains to be determined whether the aversion phenotype stimulated by caffeine is a neuronal or non-neuronal tissue response. 
Nevertheless, it is clear that expression of HSPs is critical. In summary, high-dose caffeine activated the UPR pathway and induced the expression of chaperone genes that protected worms from stress and alleviated the aversion phenotype.

Note: Supplementary information is available on the Molecules and Cells website (www.molcells.org).

\section{ACKNOWLEDGMENTS}

The nematode strains used in this study were provided by the Caenorhabditis Genetics Center. We thank the Yonsei Proteome Research Center for performing the proteomic analysis and Dr. S. Ko (Konkuk University) for technical support. This study was supported by a fund from Konkuk University in 2012 provided to Y. H. Shim.

\section{REFERENCES}

Ahn, D.H., Singaravelu, G., Lee, S., Ahnn, J., and Shim, Y.H. (2006). Functional and phenotypic relevance of differentially expressed proteins in calcineurin mutants of Caenorhabditis elegans. Proteomics 6, 1340-1350.

Benedetti, C., Haynes, C.M., Yang, Y., Harding, H.P., and Ron, D. (2006). Ubiquitin-like protein 5 positively regulates chaperone gene expression in the mitochondrial unfolded protein response. Genetics 174, 229-239.

Brenner, S. (1974). The genetics of Caenorhabditis elegans. Genetics $77,71-94$

Chen, L.W., Wu, Y., Neelakantan, N., Chong, M.F., Pan, A., and van Dam, R.M. (2014). Maternal caffeine intake during pregnancy is associated with risk of low birth weight: a systematic review and dose-response meta-analysis. BMC Med. 12, 174.

Ekwall, B., Clemedson, C., Crafoord, B., Ekwall, B., Hallander, S., Walum, E., and Bondesson, I. (1998). MEIC evaluation of acute systemic toxicity. Part V. Rodent and human toxicity data for the 50 reference chemicals. Altern. Lab. Anim. 2, 571-616.

Haynes, C.M., and Ron, D. (2010). The mitochondrial UPR - protecting organelle protein homeostasis. J. Cell Sci. 123, 38493855.

Kapulkin, W.J., Hiester, B.G., and Link, C.D. (2005). Compensatory regulation among ER chaperones in C. elegans. FEBS Lett. 579,
3063-3068.

Kawasaki, I., Jeong, M.H., Yun, Y.J., Shin, Y.K., and Shim, Y.H (2013). Cholesterol-responsive metabolic proteins are required for larval development in Caenorhabditis elegans. Mol. Cells 36, 410-416

Kuczkowski, K.M. (2009). Peripartum implications of caffeine intake in pregnancy: is there cause for concern? Rev. Esp. Anestesiol. Reanim. 56, 612-615.

Liu, Y., Samuel, B.S., Breen, P.C., and Ruvkun, G. (2014). Caenorhabditis elegans pathways that surveil and defend mitochondria Nature 508, 406-410.

Thompson KK, eds. Medication safety: a guide for health care facilities. (Bethesda, MD: American Society of Health-System Pharmacists), pp. 253-274.

Melo, J.A., and Ruvkun, G. (2012). Inactivation of conserved C. elegans genes engages pathogen- and xenobiotic-associated defenses. Cell 149, 452-466.

Mesas, A.E., Leon-Muñoz, L.M., Rodriguez-Artalejo, F., and LopezGarcia, E. (2011). The effect of coffee on blood pressure and cardiovascular disease in hypertensive individuals: a systematic review and meta-analysis. Am. J. Clin. Nutr. 94, 1113-1126.

Min, H., Kawasaki, I., Gong, J., and Shim, Y.H. (2015). Caffeine induces high expression of cyp-35A family genes and inhibits the early larval development in Caenorhabditis elegans. Mol. Cells 38, 236-242

Peltonen, J., Aarnio, V., Heikkinen, L., Lakso, M., and Wong, G. (2013). Chronic ethanol exposure increases cytochrome P-450 and decreases activated in blocked unfolded protein response gene family transcripts in Caenorhabditis elegans. J. Biochem. Mol. Toxicol. 27, 219-228.

Sutphin, G.L., Bishop, E., Yanos, M.E., Moller, R.M., and Kaeberlein M. (2012). Caffeine extends life span, improves healthspan, and delays age-associated pathology in Caenorhabditis elegans. Longev. Healthspan. 1, 1:9.

Vilarim, M.M., Rocha Araujo, D.M., and Nardi, A.E. (2011). Caffeine challenge test and panic disorder: a systematic literature review. Expert. Rev. Neurother. 11, 1185-1195.

Weinberg, B.A., and Bealer, B.K. (2002). The caffeine advantage, ed. (NY, USA:The Free press).

Yoneda, T., Benedetti, C., Urano, F., Clark, S.G., Harding, H.P., and Ron, D. (2004). Compartment-specific perturbation of protein handling activates genes encoding mitochondrial chaperones. $\mathrm{J}$. Cell Sci. 117, 4055-4066. 